\title{
ULTRASTRUCTURAL FEATURES OF HERPESVIRUS-INDUCED AORTIC INTIMA DAMAGE IN MICE FED THE HIGH-FAT DIET
}

DOI: 10.36740/WLek202003117

\author{
Nataliia S. Turchyna', Serhii I. Savosko', Tetiana M. Cherenko', Svitlana L. Ribalko², Daria B. Starosyla ${ }^{2}$ \\ 'BOGOMOLETS NATIONAL MEDICAL UNIVERSITY, KYIV, UKRAINE \\ 2L.V. GROMASHEVSKY INSTITUTE OF EPIDEMIOLOGY AND INFECTIOUS DISEASES, NAMS OF UKRAINE, KYIV, UKRAINE
}

\begin{abstract}
The aim: To study the effect of a high-fat diet (HFD) on the structural changes in the aortic intima in intact and HSV-1-infected mice using Scanning electron microscopy (SEM) and Transmission electron microscopy (TEM).

Materials and methods: In experiments Balb/c mice were infected with the HVS-1 and fed high-fat diet and 12 weeks later aortic ultrastructure was examined by SEM and TEM methods. The animals were subdivided into four experimental groups: $1^{\text {st }}$ group - HSV-1-infected animals; $2^{\text {nd }}-$ animals consuming high-fat diet (HFD); $3^{\text {rd }}-$ infected animals that were subsequently consuming a high-fat diet (HSV / HFD); $4^{\text {th }}$ - animals consuming a high-fat diet that were subsequently infected with HSV-1 (HFD / HSV) $(n=6)$; and control group - intact animals.

Results: HVS-1 impaired ultrastructural changes in aorta greater than high-fat diet and HVS-1 alone (higher density of lipid inclusions in the subendothelial space, necrosis of endothelial cells), and infection of mice after high-fat diet ended 100\% mortality. The formation of atheroma in the aortic wall during HFD was not detected, but the initiative manifestations of atherogenesis have been identified and restricted in the aortic intima. These structural changes included lipid inclusions in the subendothelial space, cell damage and destruction, which lead to an increase cellular detritus in the $3^{\text {rd }}$ (HSV / HFD) group.

Conclusions: HSV infection potentiates the accumulation of lipid inclusions in the aortic intima during a HFD, facilitates infection and contributes to the development of acute infection.
\end{abstract}

KEY WORDS: aorta, high-fat diet, HVS-1, atherogenesis, electron microscopy

Wiad Lek. 2020;73(3):498-503

\section{INTRODUCTION}

Hyperlipidemia and hypercholesterolemia are major biochemical risk factors for atherogenesis, which ultimately contribute to atherothrombosis with severe consequences. Currently, there is a small number of research articles dedicated to the structural changes in the aorta caused by hyperlipidemia and atherosclerosis as well as HSV-induced atherosclerosis [1]. Even less information is available on morphological studies of the aorta affected by a viral infection associated with atherogenesis. HSV and CMV were detected in $40 \%$ of the examined autopsy samples of the aorta and coronary vessels [2]. In experiments, the effect of HSV-infection on atherosclerosis has been studied fragmentarily, but nevertheless they demonstrate the formation of atheroma in mice with murine gammaherpesvirus-68 (MHV-68) and its progression within 24 weeks compared to the control group [3]. Murine CMV (MCMV) increases the incidence rate of atherothrombosis in apoE-knockout mice by $84 \%$, whereas Chlamydia pneumoniae (Cpn) are not associated with an increased rate of the disease [4]. Morphological signs of atherosclerosis are described in the aortic sinus in apoE-knockout mice [5].The fact that HSV-1-induced atherothrombosis does not reach the values of atheroma is linked to the development of systemic infection, even though HSV-infection promotes thrombosis and increases the risk of atherothrombosis [6].
It is currently widely recognized that any viral infection that damages endothelial cells of blood vessels can be reliably associated with the risk of vascular disease [7]. Biochemical analysis revealed that content of free and esterified cholesterol was higherin the aortic tissue specimens of CMV-infected animals with normocholesterolemia than in the models of non-infected animals. Thus, according to Fabricant C., herpesvirus infection contributed to the accumulation of lipids in the aorta [8], and previous immunization prevented atherosclerotic and fibroproliferative changes in the aortic wall [9] due to decreased activity of cytoplasmic cholesterol esterase in animals [10]. The findings were consistent with accumulation of saturated cholesterol and triacylglycerol due to impaired hydrolysis observed in the HSV-1-infected smooth muscle cells in humans [11]. The scholars describe the smooth muscle cells involved in the active synthesis of extracellular matrix of the connective tissue, such as glycosaminoglycans, collagen and elastin [12]. It is believed that the described cells originate from the media, but penetrate into the intima through the damaged inner elastic membrane of the aorta, and these altering changes are an early manifestation of atherogenesis in the intima of hypercholesterolemic animals [13].

Our hypothesis is that previous viral infection induces structural and functional changes in the tunica intima of 
blood vessels that may promote the accumulation of lipid inclusions in the aortic intima in animals consuming a high-fat and high-cholesterol diet.

\section{THE AIM}

To study the effect of a high-fat diet (HFD) on the structural changes in the aortic intima in intact and HSV-1-infected mice using Scanning electron microscopy (SEM) and Transmission electron microscopy (TEM).

\section{MATERIALS AND METHODS}

$\mathrm{Balb} / \mathrm{c}$ mice were used in the experiments considered here. The study included 5 groups of the murine aortic tissue samples:

Control group $(\mathrm{n}=3)$ - intact animals;

Group 1 - HSV-1-infected animals $(\mathrm{n}=4)$;

Group 2 - animals consuming high-fat diet (HFD) $(n=6)$; Group 3 - HSV-1-infected animals that were subsequently consuming a high-fat diet (HSV / HFD) $(\mathrm{n}=6)$;

Group 4 - animals consuming a high-fat diet that were subsequently infected with HSV-1 (HFD / HSV) $(n=6)$. The animals in the 1st group were infected with HSV-1 in L.V. Gromashevsky Institute of Epidemiology and Infectious Diseases, NAMS of Ukraine (Head of the Laboratory of Experimental Chemotherapy of Viral Infections, MD, Prof. S.L. Rybalko).

In a 12 -week experiment, the animals in the $2^{\text {nd }}$ group received the food with cholesterol, animal and vegetable fats. $100 \mathrm{~g}$ of food contained 30-45 $\mathrm{g}$ of heat-treated animal fats (pork fat / lard, butter); 15-20 g of heat-treated vegetable fats (margarine); 1.5-2 $\mathrm{g}$ of cholesterol; $1 \mathrm{~g}$ of calcium carbonate $\left(\mathrm{CaCO}_{3}\right) ; 10 \mathrm{mg}$ (in terms of daily intake for animals - $10 \mathrm{mg} / \mathrm{kg}$ ) of Mercazolyl (1-methyl 2-mercaptoimidazole with potato starch, Calcium stearate, refined sugar, talcum), 32-52 g of standard granulated animal feed.

The animals in the 3rd group were infected with HSV-1 and then received a high-fat diet as above.

The animals in the $4^{\text {th }}$ group received a high-fat diet and then were infected with HSV-1 as above.

After 12 weeks of the experiment, all animals were withdrawn from it by decapitation. After decapitation, SEM and TEM were used in the study.

SEM was used to study: 1) changes in the intima of blood vessels (surface, integrity of the endothelial layer, erythrocytes and leukocytes adhesion, thrombosis);2) cellular and non-cellular entities that affect the condition and structural integrity of the intima. SEM is an effective method that facilitates the detection of any changes in the surface of the inner layer of the aorta and makes it possible to reveal any structural lesions on a larger area compared to TEM [11]. TEM was used to study dystrophic changes involving 1) intimal endothelial cells (necrosis, vacuolisation), 2) subendothelial zone (collagen fibers, lipid inclusions); 3) smooth muscle cells.

The aorta was selected for SEM and TEM. For SEM, the aortic tissue fragments of $1-2 \mathrm{~mm}$ in size were fixed in $10 \%$ phosphate buffered (PBS) formalin solution ( $\mathrm{pH}=$
7.4). After fixation, the specimens were washed with PBS, dehydrated in ethanol, dried under a critical point of $\mathrm{CO} 2$. The dried samples were coated with gold in a thickness of $15 \mathrm{~nm}$ using the Gatan 682 PECS device. The specimens were examined under an electron microscope Tescan Mira 3 LMU (Czech Republic) in scan mode. For TEM, the aortic tissue fragments of $0.3-0.5 \mathrm{~mm}$ in size were fixed in $2.5 \%$ glutaraldehyde solution on PBS with further fixation in $1 \%$ buffered osmium tetroxide solution (OsO4); dehydration in ethanol $(70 \%, 80 \%, 90 \%, 100 \%)$ and acetone; impregnated and embedded in a mixture of epoxy resins (Epon 812, Araldite 502). The Reichert ultramicrotome was used to cut the epoxy blocks to produce ultra-thin slices of material for study. The ultra-thin sections were contrasted in $2 \%$ solution of uranyl acetate and lead citrate. The material was explored and photographed under an electron microscope Tescan Mira 3 LMU (Czech Republic) in illumination mode. TEM was used to investigate the ultrastructural changes in endothelial cells, extracellular matrix, and smooth muscle cells. The use of SEM ensured the investigation of the aortic intimal surface as well as intimal lesions within the aorta.

The heart of HSV-1-infected mice was selected to identify the virus in vitro. Homogenates were obtained from the specimens and added to a cultured Vero cell line grown in sterile plates ( «Nunc»). Composition of the culture medium: $88 \%$ of RPMI 1640 («Sigma»), 12\% of inactivated by heating serum of the embryo of the cow («PanEco» RF) and antibiotics. Cultivation conditions: $37^{\circ} \mathrm{C}, 5 \% \mathrm{CO} 2$. Detection of the virus, reproduction in Vero cells, and passages were performed on the basis of cytopathic effect registration (CPE). Cytological signs of $\mathrm{CPE}$ - syncytia. The CPE was recorded for 6-7 days.

Statistical processing of the results was carried out using the Origin Lab program, version 8.0. The normal distribution of the results was estimated by the Kolmogorov-Smirnov criterion. The intergroup discrepancies between the groups of samples were evaluated using nonparametric Kruskal-Wallis test. The difference was estimated to be significant at $\mathrm{p}<0.05$.

\section{RESULTS}

TEM and SEM were performed to detect aortic changes in the control group, $1^{\text {st }}, 2^{\text {nd }}$ and $3^{\text {rd }}$ groups (HSV, HFD and HSV / HFD). The specimens in the 4th (HFD / HSV) group were not analyzed because the group had an absolute $(100 \% \mathrm{n}=6)$ mortality in the first 3-5 days after infection. However, the heart of each infected animal was selected for virological examination.

Low-magnification SEM images $(\times 1100-\times 1500)$ showed a longitudinal waviness of the aortic lumen in intact animals, which is physiological. In the $1^{\text {st }}(\mathrm{HSV}), 2^{\text {nd }}(\mathrm{HFD})$ and $3^{\text {rd }}$ (HSV / HFD) groups, the surface had the same physiological waviness that correlates with the presence of the inner elastic membrane in the aorta and indicates its preservation.

High-magnification SEM images $(\times 8000-\times 13000)$ revealed an irregular surface with abundant cellular 


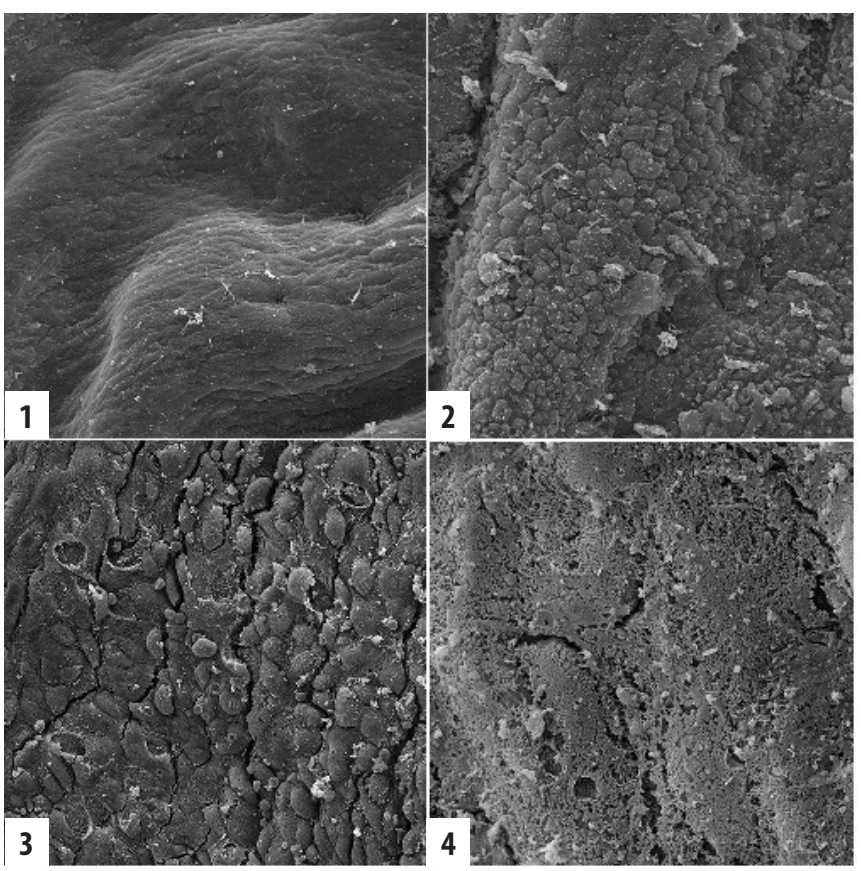

Fig. 1. Scanning electron microscope (SEM) images of the murine aortic wall. Intact and smooth surface of the aortic intima in control; swelling and protrusion of the surface of the aortic intimal cells in the $1^{\text {st }}$ (HSV) and $2^{\text {nd }}$ (HFD) groups; disrupted and coagulated intimal cells in the $3^{\text {rd }}$ (HSV / HFD) group. Note: 1$)$ control $(\times 10500) ; 2) 2^{\text {nd }}(H F D)$ group $(\times 12700)$; 3) $1^{\text {st }}(\mathrm{HSV})$ group $\left.(\times 9210) ; 4\right) 3^{\text {rd }}(\mathrm{HSV} / \mathrm{HFD})$ group $(\times 8250)$.

protrusions in the $2^{\text {nd }}$ (HFD) group (Fig. 1). This is due to edema and elevation of endothelial cells, their nuclear zone, swelling of cytoplasm above the basal membrane. The accumulation of extracellular matrix derivates and necrotic cells in the subendothelial space is not excluded.

In the $1^{\text {st }}$ (HSV) and $3^{\text {rd }}$ (HSV / HFD) groups, SEM showed adhesion of leukocytes on the surface of the aortic intima in all areas. Additionally, TEM registered leukocytes in the subendothelial zone, which can be explained by their penetration through the endothelial layer. There were no gaps in the endothelium and platelet adhesion detected in the $1^{\text {st }}(\mathrm{HSV})$ and $2^{\text {nd }}(\mathrm{HFD})$ groups, whereas in the $3^{\text {rd }}$ (HSV / HFD) group, we recorded necrotized endothelial cells, signs of coagulation and loss of intimal integrity.

The surface of the intima had additional signs of destructive changes in the form of fibrous structures that differed from the intact aorta. Those were damaged endothelial cells and most likely coagulated proteins. Such changes were more prominent in the $3^{\text {rd }}$ (HSV / HFD) than in the $1^{\text {st }}$ (HSV) group (Fig. 1).

On the surface of the intima in the $2^{\text {nd }}$ (HFD) group, erythrocyte adhesion and coagulation products were observed, but leukocytes were found less frequently. All groups had focal zones of disorganization and loss of endothelial lining (disorganization), which distinguished experimental samples from intact aortic specimens. According to SEM, erythrocyte adhesion and endothelial cell edema, seen as focal protrusions on the surface, were denser in the $2^{\text {nd }}(\mathrm{HFD})$ than in the $1^{\text {st }}(\mathrm{HSV})$ and $3^{\text {rd }}$
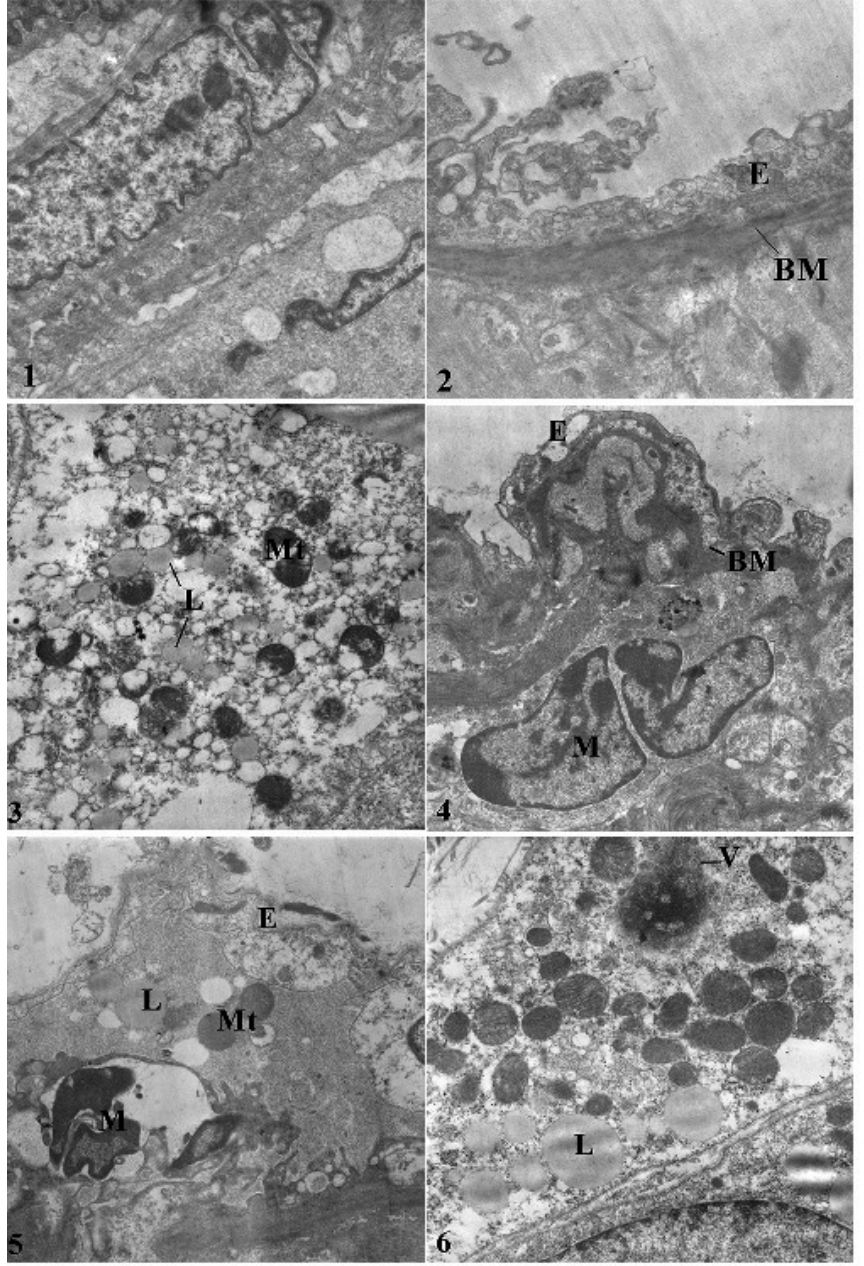

Fig. 2. Transmission electron microscope (TEM) images of endothelial cells and smooth muscle cells in the murine aorta. 1) control group: intact smooth musclecell $($ TEM,$\times 15600) ; 2) 2^{\text {nd }}(H F D)$ group: destruction and desquamation of endothelial cells in the aortic lumen $(\times 15600) ; 3) 2^{\text {nd }}($ HFD) group: phagocytosis of lipid inclusions by a macrophage («foam cell») $(\times 15600) ; 4) 1^{\text {st }}$ group (HSV): altered contour of the inner layer, vesicles in endothelial cells, monocyte with phagosomes in the subendothelial region $(\times 15600) ; 5) 3^{\text {rd }}$ (HSV / HFD) group: destruction and desquamation of endothelium, macrophages in the subendothelial space $(\times 15600) ; 6) 3^{\text {rd }}$ (HSV / HFD) group: lipid inclusions and virions in the cytoplasm of the cells in the muscle membrane $(\times 15600)$. E endothelial layer; $\mathrm{M}$ - monocyte / macrophages; $\mathrm{BL}$ - basal lamina; $\mathrm{V}$ - virions; $\mathrm{L}$ - lipid inclusions; $\mathrm{Mt}$ - mitochondria.

(HSV / HFD) groups (unfortunately, quantitative analysis is impossible). Damage and loss of endothelial cells in the intima were more pronounced in the $1^{\text {st }}$ (HSV) and $3^{\text {rd }}$ (HSV / HFD) groups than in the $2^{\text {nd }}$ (HFD) group due to the presence of a viral infection, which can explain more severe necrotic and lytic changes in endothelial cells (with the formation of focal regions, where endothelium is irregular or absent).

TEM was conducted to evaluate the following structural elements of the aortic wall: 1) endothelial layer; 2) subendothelial zone; 3 ) muscle membrane. In the control group, the aortic intima was unchanged: the nuclei of endothelial cells had a different distribution of chromatin, cytoplasmic 
Table I. Results of quantitative measurements in comparison groups (Me [Q1-03])

\begin{tabular}{cccc}
\hline Group & $\begin{array}{c}\text { Infectious titer, } \\
\text { Ig CPE 50 }\end{array}$ & $\begin{array}{c}\text { Diameter of lipid inclusions, } \\
\text { micron }\end{array}$ & $\begin{array}{c}\text { Diameter of atypical vesicles in } \\
\text { endothelial cells, micron }\end{array}$ \\
\hline Control & - & - & - \\
\hline Group 1 (HFD) & - & $0,48[0,30-0,68]$ & $0,38[0,23-0,47]$ \\
\hline Group 2 (HSV) & $1[1-2]$ & - & $0,29[0,19-0,38]$ \\
\hline Group 3 (HSV/HFD) & $2[1-3]$ & $0,66[0,46-0,98]^{*}$ & $0,68[0,43-0,67]^{*} \#$ \\
\hline Group 4 (HFD/HSV) & $3,5[2,75-4] \#$ & NA & NA \\
\hline
\end{tabular}

Note: * significant difference with the $2^{\text {nd }}(\mathrm{HFD})$ group;

\# - significant difference with the ${ }^{\text {st }}$ (HSV) group; NA - not analyzed

pinocytotic vesicles; in smooth muscle cells, intact organelles (ribosomes, vesicles, mitochondria, endoplasmic reticulum) were found (Fig. 2).

In the $2^{\text {nd }}(\mathrm{HFD})$ group, SEM showed organelle reduction, edema and destruction of mitochondria, deformation of the nuclei in endothelial cells. Endothelium atypically protrudes into the lumen of vessel and separates the derivates of cell destruction into the aortic lumen, which explains the appearance of the altered intimal contour. In some cells pinocytotic vesicles were found (the evidence of partial preservation of transendothelial transport). In the endothelium cytoplasm, there were detected atypical vesicles, most likely formed as a result of swelling of membrane organelles, in particular mitochondria. Their diameter was evaluated morphometrically (Table 1 ).

The inner elastic membrane of the aorta was structurally preserved. In the subendothelial zone of the aorta, lipid inclusions were detected. The diameter of lipid inclusions in macrophages was in the range of $150-230 \mathrm{~nm}$, and in the extracellular matrix it even reached 4 microns (Table I).

Lipid deposition contributed to the transformation of cholesterol-engorged macrophages into so-called «foam cells», destruction of individual cells and the appearance of cell free zones. Some cells were in a state of total destruction.

The morphological organization and the integrity of the murine aortic muscle membrane were preserved in the $2^{\text {nd }}$ (HFD) group, even though some changes in smooth muscle cells were found: 1) edema and destruction of cristae of mitochondria, 2) appearance of atypical vesicles in the cytoplasm, and 3) reduction of organelles and myofibrils. In some cells, accumulation of secondary lysosomes was observed. These changes indicate a secondary metabolic injury.

In endothelial cells of the 1st (HSV) group, the following changes were recorded: 1) cytoplasmic edema; 2) lysis of organelles; 3 ) atypical vesicles, which can be considered as a consequence of lytic changes and fusion of membrane organelles. In this case, the integrity of the endothelial layer was preserved. Under the endothelium, the basal membrane, internal elastic membrane, and subendothelial layer of the inner aortic membrane were observed. In subendothelial zone, the following changes were revealed: 1) macrophages; 2) diapedesis of some blood corpuscles, 3) decrease in the density of collagen fibers. The revealed changes indicate a decrease in: 1 ) the barrier function of endothelial cells, 2) the infiltration of leukocytes; 3 ) the reorganization of paravasal intercellular space, which is a manifestation of the inflammatory reaction and is a qualitative morphological distinction from the 2nd (HFD) group. Under the elastic membrane, smooth muscle cells of the aortic media were found without any significant structural changes.

The $3^{\text {rd }}$ (HSV / HFD) group had progressive structural changes, as described in the $1^{\text {st }}$ (HFD) group. Changes in the endothelium in the form of: 1) edema and destruction of organelles, 2) the appearance of atypical vesicles of greater diameter; 3 ) necrosis of cells with desquamation and endothelial damage. During SEM these changes were seen as: 1) sites of destruction, coagulation; 2 ) loss of the endothelial cells (Table 1). The increase in the density and diameter of atypical vesicles by $57.8 \%(\mathrm{P}=0.05)$ indicates the progression of dystrophic changes in the aortic endothelial cells in the $3^{\text {rd }}$ (HSV / HFD) group compared to the $2^{\text {nd }}(\mathrm{HFD})$ group. Accumulation of virions (diameter $120-150 \mathrm{~nm}$ ) in injured cell was detected in the one sample.

In the subendothelial layer, the following changes were also revealed: 1) accumulation of lipid inclusions, 2) destructive changes, 3 ) reorganization of the extracellular matrix and infiltration of macrophages. The diameter of lipid inclusions was $37.5 \%$ higher $(\mathrm{P}<0.05)$ compared to the $2^{\text {nd }}$ (HFD) group. The density of macrophages with "foamy» cytoplasm also increased, and the reorganization of collagen fibers can be explained by phagocytic activity of macrophages and their participation in remodeling of extracellular matrix. This indicates a higher degree of structural damage to the aorta in the $3^{\text {rd }}$ (HSV / HFD) group compared to the $2^{\text {nd }}(\mathrm{HFD})$ group, that is HSV1 potentiates their development.

The morphological integrity of the muscle membrane was preserved. But, whereas in the $2^{\text {nd }}$ (HFD) group only atypical vesicles were detected in smooth muscle cells, in the $3^{\text {rd }}$ (HSV / HFD) group, perinuclear edema of the nucleus was additionally revealed. This indicates that the presence of HSV infection potentiates the development of ultrastructural changes in the aortic media. Changes in smooth muscle cells are nonspecific and most likely related to metabolic disorders caused by a 12-week consumption of a high-fat diet in the $2^{\text {nd }}$ (HFD) group.

TEM demonstrated that injured aortic intima had insufficient number of ultrastructural markers to identify and verify the damage factor. Lipid inclusions occurred 
in the $2^{\text {nd }}(\mathrm{HFD})$ and $3^{\text {rd }}$ (HSV / HFD) groups, and signs of viral damage to endothelial cells (lamellar cells, single virions) were detected only in one samples from the $3^{\text {rd }}$ (HSV / HFD) group.

In vitro studies in all cardiac homogenates confirmed the presence of HSV-1, and the level of reproduction was estimated according to CPE (Table 1). Although there was no significant difference between the 1 st (HSV) and the $3^{\text {rd }}$ (HSV / HFD) groups, fatal cases of another model of the $4^{\text {th }}$ group (HFD / HSV) caused significantly higher CPE in the hearts. It is clear that the term of infection period is different, but the absolute lethality of mice that received a high-fat diet for 12 weeks and then were infected with HSV-1, gives reason to think about a certain direct and inverse relationship in the study.

\section{DISCUSSION}

Changes in endothelial cells and extracellular matrix were more pronounced in the $3^{\text {rd }}$ (HSV / HFD) group compared to the $2^{\text {nd }}(\mathrm{HFD})$ group. The most striking changes in the aortic intima were observed as the accumulation of lipid inclusions and macrophages in the subendothelial space. The revealed changes in the subendothelial space of the aorta can be explained by: 1) migration of monocytes / macrophages from blood or regional phagocytes for the elimination of lipid inclusions in the $2^{\text {nd }}(\mathrm{HFD})$ group; 2 ) adhesion and migration of leukocytes to the vascular wall for aggressive response to HSV-1 in the $1^{\text {st }}$ (HSV) group; 3 ) lipid deposition of cellulardebris in the extracellular matrix and macrophages (lysosomal deposits) promoted by a structurally damaged endothelial layer under the influence of HSV-1 in the $3^{\text {rd }}$ (HSV / HFD) group. The lipid inclusions and necrotic cell debris were phagocyted by macrophages in subendothelial space of the $2^{\text {nd }}$ (HFD) and $3^{\text {rd }}$ (HSV / HFD) groups. However, these processes were more intense in the $3^{\text {rd }}$ (HSV / HFD) group, the density of the detected phagocytes, the number and diameter of lysosomal inclusions were also greater in this group. In the $1^{\text {st }}$ (HSV) group, macrophages were also detected, but their migration, in our opinion, can be associated with the elimination of cellular detritus. The latter is due to lytic changes in the cells that are induced by HSV-1, and the appearance of individual lymphocytes within the aortic wall in response to the infection.

In the third group (HSV / HFD), damage to the subintimal smooth muscle cells (edema and destruction of mitochondria, decrease in electron density and fibrous structures) was detected. We believe that in the $3^{\text {rd }}$ (HSV / HFD) group ultrastructural changes progress from the aortic intima and subintimal matrix to the muscular layer. The prominent signs of necrosis of smooth muscle cells in mice after a 12-week high-fat diet were not observed (the damage was manifested only by the destruction of mitochondria).

SEM, used to evaluate the surface of the aorta, also yielded some interesting results. Prior to the experiment, we made an assumption about structural abnormalities in the aortic wall in the $2^{\text {nd }}$ (HFD) group that should have been in the form of changes on inner surface of aorta, but after 12 weeks of a high-fat diet those alterations did not occur. The aortic intima had a longitudinal physiological waviness similar to that of the intact aorta of mice, as described previously [14]. But other important peculiarities were observed in infected animals. The $1^{\text {st }}(\mathrm{HSV})$ and $3^{\text {rd }}$ (HSV / HFD) groups demonstrated «abundant» adhesion of leukocytes to the aortic intima, whereas the $2^{\text {nd }}$ (HFD) group showed more intense erythrocyte adhesion and swelling of the endothelial cells. Between those cells the structural defects were seen (gaps, lysis of cells with remnants of the nuclear zone of endotheliocytes). It was found that the contours of the neighboring cells did not have complete contact. The findings obtained by means of SEM correlate with TEM results and complement them. Similar gaps are described by other authors and are apparently a nonspecific manifestation of the aortic intima damage [15]. The results obtained in our study correlate with the findings that were published earlier.

\section{CONCLUSIONS}

1. The formation of atheroma in the aortic wall during a 12-week high-fat diet, as shown in the literature, was not detected.

2. Comparative analysis of the aortic changes in mice of the $1^{\text {st }}, 2^{\text {nd }}$ and $3^{\text {rd }}$ groups (HSV, HFD and HSV / HFD) proves the initiation of structural changes in the aortic intima including phagocytosis of individual lipid inclusions in the subendothelial space in the $2^{\text {nd }}$ (HFD) group and their subsequent accumulation in cells, cell damage and destruction, which leads to an increase in the density of lipid inclusions and cellular detritus in the extracellular aortic matrix in the $3^{\text {rd }}$ (HSV / HFD) group.

3. HSV infection potentiates the accumulation of lipid inclusions in the aortic intima during a HFD, facilitates infection and contributes to the development of acute infection.

\section{REFERENCES}

1. Dahal U, Sharma D, Dahal K. An Unsettled Debate About the Potential Role of Infection in Pathogenesis of Atherosclerosis. J Clin Med Res. 2017; 9(7):547-554.

2. Yamashiroya HM, Ghosh L, Yang R et al. Herpes viridae in the coronary arteries and aorta of young trauma victims. Am J Pathol. 1988; 130(1):71-79.

3. Alber DG, Powell KL, Vallance P et al. Herpes virus infection accelerates atherosclerosis in the apolipoprotein E-deficient mouse. Circulation. 2000; 102(7):779-785.

4. Burnett MS, Gaydos CA, Madico GE et al. Atherosclerosis in apoE knockout mice infected with multiple pathogens. J Infect Dis. 2001;183(2):226-231.

5. Heestermans $M, 0$ uweneel $A B$, Hassan J et al. Predilection of low protein c-induced spontaneous atherothrombosis for the right coronary sinus in apolipoprotein E deficient mice. SciRep. 2018; 8(1):15106.

6. Wu YP, Sun DD, Wang Y et al. Herpes Simplex Virus Type 1 and Type 2 Infection increases atherosclerosis risk: evidence based on a meta-analysis. Biomed Res Int. 2016; 2016:2630865. doi: $10.1155 / 2016 / 2630865$. 
7. Motorna N, Rybalko S, Kvitnitskaya-Ryzhova T et al. Ultrastructural changes in murine liver following HSV infection and stroke. Curr Issues Pharm Med Sci. 2018;31(1):10-12.

8. Hajjar D, Fabricant C, Minick Cet al. Virus-induced atherosclerosis: herpes virus infection alters arterial cholesterol metabolism and accumulation. Am J Pathol. 1986; 122(1):62-70.

9. Fabricant C, Fabricant J, Minick CR et al. Herpesvirus induced atherosclerosis in chickens. Fed Proc, 1983;42(8):2476-2479.

10. Fabricant CG, Krook L, Gillespie J. Virus-induced cholesterol crystals. Science. 1973;181(4099):566-567.

11. Hajjar D. Herpesvirus infection prevents activation of cytoplasmic cholesterylesterase in arterial smooth muscle cells. J Biol Chem. 1986; 261(17):7611-7614.

12. Haudenschild CC, Prescott MF, Chobanian AV. Aortic endothelial and subendothelial cells in experimental hypertension and aging. Hypertension. 1981; 3(3 Pt 2):1148-153.

13. KwonHM,SangiorgiG,SpagnoliLGetal.Experimental hypercholesterolemia induces ultrastructural changes in the internal elastic lamina of porcine coronary arteries. Atherosclerosis. 1998;139(2):283-289.

14. Mello JM, Orsi AM, Padovani CR et al. Some segmental structural features of the aortic wall of domestic chicken (Gallusdomesticus). Braz JVet Res Anim Sci. 2003;40(3):163-169.

15. Maeda N, Hagihara H, Nakata Y et al. Aortic wall damage in mice unable to synthesize ascorbic acid. Proc Natl Acad Sci USA. 2000;97(2):841-846.

Research work performed by Bogomolets National Medical University: "Study of the features and consequences of stroke in patients of different age groups based on the genetic and infectious factors and comorbid pathology"

\section{ORCID and contributionship:}

Nataliia S. Turchyna - 0000-0002-9992-4929 A, B, D

Serhii I. Savosko - 0000-0001-5145-2195 C,D,F

Tatiana M. Cherenko - 0000-0003-0867-5929 A, B, E, F

Daria B. Starosyla - 0000-0003-0210-2361 A, B, E, F

Svitlana L. Ribalko -0000-0002-1913-1380 A, B, E, F

\section{Conflict of interest:}

The Authors declare no conflict of interest.

\section{CORRESPONDING AUTHOR}

\section{Serhii I. Savosko}

Bogomolets National Medical University, Tarasa Shevchenko Blvd, 13, Kyiv, 01601, Ukraine tel: +380976278086

e-mail:s.i.savosko@gmail.com

Received: 17.01 .2020

Accepted: 05.03 .2020

A - Work concept and design, B - Data collection and analysis, C - Responsibility for statistical analysis, D - Writing the article, $\mathbf{E}$ - Critical review, $\mathbf{F}$ - Final approval of the article 